\title{
Nano-enabled drug delivery research: A scientometric assessment of Indian pulblications during 1995-2018
}

\author{
K. K. Mueen Ahmed, B. M. Gupta ${ }^{1}$
}

Phcog.Net, Bengaluru, Karnataka, ${ }^{1}$ Formerly with CSIR-National Institute of Science, Technology and Development Studies, New Delhi, India

\begin{abstract}
Aim: The present study is aimed on to perform Scientometric assessment of global publications output of research on nano-enabled drug delivery (NEDD) during 1995-2018.

Materials and Methods: A quantitative and qualitative description of NEDD research in India is presented here based on measures such as growth rate, global publications share, share of international collaborative papers, citations per paper, and highly cited papers. It was examined India's research output on NEDD on a series of bibliometric indicators.

Results: India published a total of 5897 publications in 24 years during 1995-2018, registering an average annual growth rate of $42.99 \%$, citation impact of 22.90 citations per paper, global publication share of $11.11 \%$, and international collaborative publication share of $20.35 \%$. Profiles global publication output and share of top 10 most productive countries in NEDD research, 20 most productive Indian organizations and 20 most productive Indian authors on a series of indicators including publications output, number of citations, the relative citation index, citations per paper, h-index, and share of international collaborative papers during 1995-2018.

Conclusion: This study describes the scattering of research output of India across source journals and distribution of research by subject areas that intersect with NEDD research. It suggests the need for India to develop specific plans of action and ensure its implementation at the national level and also prioritize national goals for NEDD research.
\end{abstract}

Keywords: Bibliometrics, Indian publications, nano-enabled drug delivery, Scientometrics

Address for correspondence: Dr. K. K. Mueen Ahmed, Phcog.Net, No. 9, Wheelers Road Extn, St. Thomas Town, Bengaluru, Karnataka, India. E-mail: mueen.ahmed@gmail.com

\section{INTRODUCTION}

Drug delivery research has grown rapidly over the past two decades and has enabled drug development by designing suitable delivery systems that improve efficacy, lower dosing frequency, and encourage patient convenience and compliance..$^{[1]}$ Drug delivery is an important issue, especially with a new generation of therapeutics, which are either unstable in the biological environment, have

\begin{tabular}{|l|l|}
\hline \multicolumn{2}{|c|}{ Access this article online } \\
\hline Quick Response Code: & Website: \\
\hline & www.jpionline.org \\
\cline { 2 - 3 } & \\
\hline
\end{tabular}

poor transport properties across biological membranes, are insoluble in water, or have very low bioavailability. Within the last 10 years, nano-enabled drug delivery (NEDD) has drawn the attention of research and industry areas, as a key nanotechnology. Nanoparticulate drug-delivery vehicles have been developed using various nanomaterials and components. Such systems have the ability to encapsulate and carry the payload (therapeutics) and penetrate through biological membranes to deliver that payload to

This is an open access journal, and articles are distributed under the terms of the Creative Commons Attribution-NonCommercial-ShareAlike 4.0 License, which allows others to remix, tweak, and build upon the work non-commercially, as long as appropriate credit is given and the new creations are licensed under the identical terms.

For reprints contact: reprints@medknow.com

How to cite this article: Mueen Ahmed KK, Gupta BM. Nano-enabled drug delivery research: A scientometric assessment of Indian publications during 1995-2018. Int J Pharma Investig 2018;8:182-91. 
specific target disease sites. The outstanding advantage of NEDD is that the applicable nanoparticles can keep the pharmaceutical well protected from degradation and prolong the exposure of the pharmaceutical through controlled release. Thus, NEDD provides a novel approach to medical therapy, including treatment of chronic diseases and genetic disorders. ${ }^{[2]}$

In the last decade, several drug-delivery technologies have emerged for the development of nanoscale drug delivery devices. The enormous growth of nanotechnology paved ways for the development of advanced drug delivery systems having ultraprecision and control over the release of drugs. Different strategies are adopted to ensure targeted and controlled drug delivery, such as encapsulation, biomarkers, and artificial nanocarriers. Extensive types of nanocarriers (such as nanoparticles, nanowires, nanocages, nanoshells, and nanodiamonds) composed of an assortment of different sizes, shapes, and materials and with various chemical and surface properties, have already been constructed. These nanocarriers normally consist of biological materials or chemically synthesized materials or combination of both, such as dendrimers, protein, lipid, chitosan, lactic acid, polymers, magnetic, fullerenes, quantum dots, carbon, and silica are being developed for various drug-delivery applications..$^{[3]}$

\section{Literature review}

Only a few scientometric studies are available on drug delivery technology, particularly the NEDD. Robert et al. ${ }^{[4]}$ studied the growth of the drug delivery literature published during 1974-2015 from the journals indexed in the Science Citation Index Expanded Database. The growth of publications on drug delivery paralleled the total scientific publications for three decades (1974-2003); however, from 2004 to 2015, it exploded fourfold, while the total increased only 1.75-fold. Industrialized countries (the USA, UK, Germany, Japan, Italy, France, and Canada) were the most prolific during the first decades, but in 2014-2015, China, India, and South Korea ranked $1^{\text {st }}, 3^{\text {rd }}$, and $4^{\text {th }}$, respectively, among the productive countries. The number of participating countries increased fivefold (from 19 to 96). During the last 15 years, the journals targeted by drug delivery research increased nearly 2.4-fold (416-1001) and three journals (Journal of Controlled Release, Advanced Drug Delivery Reviews and International Journal of Pharmaceutics) published nearly one-fifth of the drug delivery research in 2014-2015.

Zhou et al. ${ }^{[5]}$ described the process of how to derive NEDD-related information from global research and patent databases in an effort to perform a comprehensive global analysis of research trends and directions, along with collaborative patterns. Yen-Chun et al. ${ }^{[6]}$ studied the scientific literature of nanoparticle drug delivery technologies between 2005 and 2014 was reviewed. The visualized cocitation network of its knowledge domain was characterized in terms of thematic concentrations of cocited references and emerging trends of surging keywords and citations to references through a scientometric review. The combined dataset of 25,171 bibliographic records was constructed through topic search and citation expansion to ensure adequate coverage of the field. This study not only facilitated the connection of authors and research themes in the NEDD community, but also demonstrated how research interests and trends evolve over time, which greatly contributes to our understanding of the NEDD knowledge domains. Huang et al. ${ }^{[2]}$ used bibliometric and social network analyses, at country, institution, and individual levels, to explore the patterns of scientific networking for a key nanoarea - NEDD. The data for this research come from a global compilation of research publication information on NEDD directed at brain cancer. The authors derive a set of indicators that address multiple facets of research collaboration and knowledge transfer patterns. The study results show that: (i) international cooperation is increasing, but networking characteristics change over time; (ii) highly productive institutions also lead in influence, as measured by citation to their work, with American institutes leading; and (iii) research collaboration is dominated by local relationships, with interesting information available from authorship patterns that go well beyond journal impact factors.

\section{MATERIALS AND METHODS}

\section{Objectives}

NEDD systems are rapidly emerging as a key area for nanotechnology application. Understanding the status and developmental prospects of this area around the world and India, in particular, is important to determine research priorities and to evaluate and direct progress. Global research publication and patent databases provide a reservoir of information that can be tapped to provide intelligence for such needs. The present study is aimed at making a scientometric assessment of India's publications in NEDD systems, indexed in the Scopus database during 1995-2018. The specific objectives are to study:

- The growth and distribution of world and top 10 countries publications

- Indian literature on NEDD research, with particular reference to its publication output, its global publication share, and citation impact 
- International collaboration and identification of major collaborators with India

- The distribution of India's publication output by broad subject areas and the identification of significant keywords

- The scientometric profile of 10 most productive countries, 20 most productive Indian organizations, and 20 most productive Indian authors

- The leading medium of communication in India and the bibliographic characteristics of highly cited publications by India on NEDD research.

\section{Methodology}

The global publications and of world and of top 10 countries in NEDD research were sourced from the Scopus international database (http://www.scopus.com) using a keywords, "drug delivery" and "Nano*" for the years 1995-2018. The "Article title" or "Keyword" (As shown in the search string below) tags were searched for the keyword restricting the hit to the period 1995-2018 in "date range tag." This statement became the main search string. The main search string was further restricted to 10 countries one by one in "country tag" for obtaining publication information of these countries (as shown below for India). On further restricting India's main search string (as provided in analytical functions of Scopus database) by "subject area tag," "country tag," "source title tag," "journal title name" and "affiliation tag," statistics on distribution of publications by subject, collaborating countries, author-wise, organization-wise, and journal-wise were citation data was obtained from the date of publication till December 22, 2018.

- KEY ("drug delivery" and "Nano*”) OR TITLE ("drug delivery" and "Nano*") AND PUBYEAR > 1994 AND PUBYEAR < 2019

- KEY ("drug delivery" and "Nano*") OR TITLE ("drug delivery" and "Nano*") AND PUBYEAR > 1994 AND PUBYEAR < 2019 AND (LIMIT-TO (AFFILCOUNTRY, "India”).

\section{Analysis}

NEDD research output consisting of 53,079 global and 5897 Indian publications were derived from Scopus database and studied during 24 years (1995-2018). The annual and cumulative publication output of world and India increased from 48 and 1 in the year 1995 to 95 publications in the year 2018, averaging $25.67 \%$ and $42.99 \%$ annual growth rate, respectively. The global and Indian cumulative output (computed in 12 years) in NEDD research increased from 3343 and 151 (during 1995-2006) to 49,736 and 5746 (during 2007-2018), registering $1387.77 \%$ and $3705.30 \%$ growth rates, respectively. India's global share in NEDD research averaged to $11.11 \%$ during 1995-2018, which increased from 4.52\% during 1995-2006 to $11.55 \%$ during $2007-2018$. The citation impact of Indian publications on in NEDD research averaged to 22.90 citations per publication (CPP) during 1995-2018, which declined from 150.66 CPP during 1995-2006 to 19.54 CPP during 2007-2018 [Table 1]. Of the total Indian publications output, $66.32 \%$ (3911) was published as articles, $24.42 \%$ (1440) as reviews, $4.49 \%$ (265) as book chapters, $2.63 \%$ (155) as conference papers, $0.58 \%$ (34) as editorials, $0.56 \%$ (33) as articles in press and the rest as short surveys (19 and $0.32 \%)$, books and letters (14 and $0.24 \%$ each), notes (6 and $0.10 \%$ ), erratum ( 4 and $0.07 \%)$, and retracted ( 2 and $0.03 \%$ ) during 1995-2018.

\section{International collaboration}

About 20.35\% (1200) of the total research output by India in NEDD research was published as international collaborative papers during 1995-2018. India's collaborative output increased from $14.57 \%$ during $1995-2006$ to $20.50 \%$ during 2007-2018. The 1200 international collaborative papers by India together received 34013 citations, averaging to 28.34 citations per paper. The leading foreign countries that collaborated with India in NEDD research were: the USA (33.75\% publication share), followed by Saudi

Table 1: Annual publications output by world and India on nano-enabled drug delivery research during 1995-2018

\begin{tabular}{|c|c|c|c|c|c|c|}
\hline \multirow[t]{2}{*}{ Period } & \multirow{2}{*}{$\begin{array}{l}\text { World } \\
\text { TP }\end{array}$} & \multicolumn{5}{|c|}{ India } \\
\hline & & TP & TC & CPP & $\begin{array}{c}\text { Percentage } \\
\text { TP }\end{array}$ & ICP (\%) \\
\hline 1995 & 48 & 1 & 21 & 21 & 2.08 & \\
\hline 1996 & 56 & 3 & 103 & 34.33 & 5.36 & \\
\hline 1997 & 74 & 3 & 28 & 9.33 & 4.05 & \\
\hline 1998 & 72 & 4 & 50 & 12.50 & 5.56 & \\
\hline 1999 & 106 & 2 & 48 & 24.00 & 1.89 & \\
\hline 2000 & 97 & 4 & 2853 & 713.25 & 4.12 & \\
\hline 2001 & 140 & 4 & 2882 & 720.50 & 2.86 & $1(25.00)$ \\
\hline 2002 & 211 & 5 & 316 & 63.20 & 2.37 & $0(0.00)$ \\
\hline 2003 & 284 & 10 & 1461 & 146.10 & 3.52 & $1(10.00)$ \\
\hline 2004 & 505 & 27 & 6467 & 239.52 & 5.35 & $6(22.22)$ \\
\hline 2005 & 753 & 35 & 3526 & 100.74 & 4.65 & $7(20.00)$ \\
\hline 2006 & 997 & 53 & 4994 & 94.23 & 5.32 & 7 (13.21) \\
\hline 2007 & 1286 & 82 & 7536 & 91.90 & 6.38 & $12(14.63)$ \\
\hline 2008 & 1772 & 121 & 8296 & 68.56 & 6.83 & 18 (14.88) \\
\hline 2009 & 2217 & 183 & 9422 & 51.49 & 8.25 & 35 (19.13) \\
\hline 2010 & 2863 & 298 & 17,239 & 57.85 & 10.41 & 53 (17.79) \\
\hline 2011 & 3262 & 428 & 12,057 & 28.17 & 13.12 & $70(16.36)$ \\
\hline 2012 & 3995 & 455 & 13,116 & 28.83 & 11.39 & 68 (14.95) \\
\hline 2013 & 4579 & 567 & 12,052 & 21.26 & 12.38 & 98 (17.28) \\
\hline 2014 & 5017 & 591 & 12,458 & 21.08 & 11.78 & 106 (17.94) \\
\hline 2015 & 5453 & 662 & 8644 & 13.06 & 12.14 & $143(21.60)$ \\
\hline 2016 & 5943 & 797 & 7243 & 9.09 & 13.41 & $172(21.58)$ \\
\hline 2017 & 6340 & 754 & 3255 & 4.32 & 11.89 & 189 (25.07) \\
\hline 2018 & 7009 & 808 & 976 & 1.21 & 11.53 & 214 (26.49) \\
\hline 1995-2006 & 3343 & 151 & 22,749 & 150.66 & 4.52 & 22 (14.57) \\
\hline 2007-2018 & 49,736 & 5746 & 112,294 & 19.54 & 11.55 & 1178 (20.50) \\
\hline 1995-2018 & 53,079 & 5897 & 135,043 & 22.90 & 11.11 & $1200(20.35)$ \\
\hline
\end{tabular}

TP: Total paper, TC: Total citation, CPP: Citations per paper, ICP: International collaborative paper 
Arabia (13.42\%), Malaysia and South Korea (7.58\% each), Australia (6.33\%), Japan, Canada, Germany, the UK, and South Africa (from 3.33\% to 4.67\%) during 1995-2018. India's international collaborative publications (ICP) share showed increase in Saudi Arabia, Malaysia, South Korea, the UK, South Africa, and Australia (from 1.82\% to $13.67 \%$ ), as against decrease in the USA, Canada, Germany, and Japan (from 4.51\% to $21.18 \%$ ) from 1995-2006 to 2007-2018 [Table 2].

Top productive countries contribution in global nano-enabled drug delivery research

The global NEDD research witnessed the participation of 134 countries during 1995-2018, of which 80 countries contributed $1-50$ papers each, 7 countries $51-100$ papers each, 24 countries $101-500$ papers each, 9 countries 501-1000 papers each, 12 countries $1001-5000$ papers each, and 2 countries 12,092-13,244 papers each. However, the top 10 countries together alone accounted for $85.04 \%$ global publication share during 1995-2018, which showed increase from $82.08 \%$ during $1995-2006$ to $85.24 \%$ during 2007-2018. Individually, the top 10 countries publications ranged from 1839 to 13244 and accounted for $3.46 \%-24.95 \%$ of global publication share during 1995-2018. The USA, among top countries, accounted for the largest publication share $(24.95 \%)$, followed by China $(22.78 \%)$, India $(11.11 \%)$, Germany, the UK, South Korea, and Italy (from $4.00 \%$ to $4.58 \%$ ) and Iran, Japan, and France (from 3.46\% to 3.86\%) during 1995-2018. Four countries which showed increase in their global publication share (from $0.08 \%$ to $115.28 \%$ ) in 12 years were: China, India, Iran, and Italy, as against six countries, namely, the USA, France, Germany, Japan. The UK and South Korea showing decrease in their global publication share (from 1.27\% to 6.80\%) from 1995-2006 to 2007-2018 [Table 3].

Subject-wise distribution of Indian research on nano-enabled drug delivery research

NEDD research output from India published during 1995-2018 was classified under seven broad subjects (as defined by Scopus database) during 1995-2018. Pharmacology, toxicology, and pharmaceutics accounted for the largest publication share $(53.72 \%)$, followed by biochemistry, genetics and molecular biology (27.51\%), materials science $(25.57 \%)$, engineering $(22.01 \%)$, chemical engineering $(20.89 \%)$, medicine $(19.50 \%)$,

Table 2: Publication share of leading foreign countries in India's collaborative papers output in nano-enabled drug delivery research during 1995-2018

\begin{tabular}{|c|c|c|c|c|c|c|c|}
\hline \multirow[t]{2}{*}{ Serial number } & \multirow[t]{2}{*}{ Collaborative country } & \multicolumn{3}{|c|}{ Number of ICP } & \multicolumn{3}{|c|}{ Share of ICP } \\
\hline & & $1995-2006$ & $2007-2018$ & $1995-2018$ & $1995-2006$ & 2007-2018 & 1995-2018 \\
\hline 1 & USA & 12 & 393 & 405 & 54.55 & 33.36 & 33.75 \\
\hline 2 & Saudi Arabia & 0 & 161 & $=161$ & 0.00 & 13.67 & 13.42 \\
\hline 3 & Malaysia & 0 & 91 & 91 & 0.00 & 7.72 & 7.58 \\
\hline 4 & South Korea & 0 & 91 & 91 & 0.00 & 7.72 & 7.58 \\
\hline 5 & Australia & 1 & 75 & 76 & 4.55 & 6.37 & 6.33 \\
\hline 6 & Japan & 2 & 54 & 56 & 9.09 & 4.58 & 4.67 \\
\hline 7 & Canada & 4 & 50 & 54 & 18.18 & 4.24 & 4.50 \\
\hline 8 & Germany & 2 & 48 & 50 & 9.09 & 4.07 & 4.17 \\
\hline 9 & UK & 0 & 48 & 48 & 0.00 & 4.07 & 4.00 \\
\hline 10 & South Africa & 0 & 40 & 40 & 0.00 & 3.40 & 3.33 \\
\hline Indian total & & 22 & 1178 & 1200 & & & \\
\hline
\end{tabular}

ICP: International collaborative paper

Table 3: Publication output and global publication share (percentage) of top 10 most productive countries in nano-enabled drug delivery research during 1995-2018

\begin{tabular}{|c|c|c|c|c|c|c|c|}
\hline \multirow[t]{2}{*}{ Serial number } & \multirow[t]{2}{*}{ Name of the country } & \multicolumn{3}{|c|}{ Number of papers } & \multicolumn{3}{|c|}{ Share of papers } \\
\hline & & $1995-2006$ & $2007-2018$ & $1995-2018$ & 1995-2006 & $2007-2018$ & 1995-2018 \\
\hline 1 & USA & 1047 & 12,197 & 13,244 & 31.32 & 24.52 & 24.95 \\
\hline 2 & China & 283 & 11,809 & 12,092 & 8.47 & 23.74 & 22.78 \\
\hline 3 & India & 151 & 5746 & 5897 & 4.52 & 11.55 & 11.11 \\
\hline 4 & Germany & 284 & 2147 & 2431 & 8.50 & 4.32 & 4.58 \\
\hline 5 & UK & 206 & 2111 & 2317 & 6.16 & 4.24 & 4.37 \\
\hline 6 & South Korea & 122 & 1183 & 1305 & 3.65 & 2.38 & 2.46 \\
\hline 7 & Italy & 131 & 1990 & 2121 & 3.92 & 4.00 & 4.00 \\
\hline 8 & Iran & 9 & 2038 & 2047 & 0.27 & 4.10 & 3.86 \\
\hline 9 & Japan & 240 & 1608 & 1848 & 7.18 & 3.23 & 3.48 \\
\hline 10 & France & 271 & 1568 & 1839 & 8.11 & 3.15 & 3.46 \\
\hline \multicolumn{2}{|l|}{ Total } & 2744 & 42,397 & 45,141 & 82.08 & 85.24 & 85.04 \\
\hline \multicolumn{2}{|c|}{ World total } & 3343 & 49,736 & 53,079 & & & \\
\hline \multicolumn{2}{|c|}{ Share of top 10 countries in world total } & 82.08 & 85.24 & 85.04 & & & \\
\hline
\end{tabular}


and chemistry (19.31\%) during 1995-2018. The activity index showed increase in publication activity in engineering, materials science, chemical engineering, biochemistry, genetics and molecular biology, medicine, and chemistry (from 14.66 to 59.40), as against decrease in pharmacology, toxicology, and pharmaceutics by 21.35 from 1995-2006 to 2007-2018. The average value of activity index is 100 . Chemical engineering registered the highest citation per paper (28.43), followed by pharmacology, toxicology and pharmaceutics (24.08), biochemistry, genetics and molecular biology (27.51), chemistry (27.11), materials science (24.72), medicine (19.12), and engineering (18.05) during 2008-2017 [Table 4].

\section{Significant keywords}

Around 108 significant keywords have been identified from the literature which through light on the research trends in NEDD research, including different types of nanocarriers along with materials used, target therapies for different diseases, types of pharmaceuticals, and various medicines used. These keywords are listed in Table 5 in the decreasing order of the frequency of their occurrence in the literature during 1995-2018 [Table 5].

\section{Contribution and citation impact of top 20 most} productive Indian organizations

Six hundred and fifty-eight organizations participated in Indian NEDD research, of which 472 organizations contributed 1-10 papers each, 93 organizations 11-20 papers each, 34 organizations $21-30$ papers each, 68 organizations 21-50 papers each, 22 organizations 51-100 papers each, 10 organizations 101-200 papers each, and 3 organizations 201-278 papers each. Of the 658 participating organizations, the 20 most productive organizations in NEDD research together contributed 2360 publications (40.02\% share) and 70,329 citations (52.08\% share) in India's output during 1995-2018. Individually, the top 20 organizations contribution varied from 67 to 304 in 24 years. Only four organizations registered productivity rate above the group average of 118 per organization: Jamia
Hamdard University, Delhi (304 papers); Dr. Harisingh Gour University, Sagar, M.P. (278 papers); Panjab University, Chandigarh (169 papers); and University of Delhi (142 papers). Only ten organizations registered citation impact and Relative Citation Index above the group average of 29.80 CPP and 1.30: National Institute of Pharmaceutical Education and Research, Mohali (73.87 and 3.23); Indian Institute of Technology, Kharagpur (40.11 and 1.75); Dr. Harisingh Gour University, Sagar, M.P. (38.82 and 1.70); M.S. University of Baroda (36.18 and 1.58); Amrita Institute of Medical Sciences, Coimbatore (34.39 and 1.50); Institute of Chemical Technology, Mumbai (34.37 and 1.50); Amrita Vishwa Vidyalayam University, Kochi (33.99 and 1.48); Institute of Nuclear Medicine and Allied Sciences, Delhi (33.60 and 1.47); University of Delhi (30.39 and 1.33); and Banaras Hindu University, Varanasi (29.88 and 1.30). Ten organizations registered ICP above the group average of $21.95 \%$ : Indian Institute of Science, Bengaluru (38.81\%); University of Delhi (32.39\%), Indian Institute of Chemical Technology, Hyderabad (29.41\%); Amrita Institute of Medical Sciences, Coimbatore (29.03\%); Jamia Hamdard University, Delhi (28.29\%); Banaras Hindu University, Varanasi (27.10\%); National Institute of Pharmaceutical Education and Research, Mohali (26.73\%); Amrita Vishwa Vidyalayam University, Kochi $(25.32 \%)$; Indian Institute of Technology, Mumbai (24.11\%); and Indian Institute of Technology, BHU, Varanasi (22.34\%) during 1995-2018 [Table 6].

Contribution and citation impact top 20 most productive Indian authors

Eight hundred and eleven authors participated in Indian NEDD research, of which 743 authors contributed 1-10 papers each, 139 authors $11-20$ papers each, 47 authors $21-50$ papers each, 11 authors 51-100 papers each, and 1 author 101-109 papers. Of the 811 participating organizations, the top 20 most productive authors contributed 37-109 papers each and together they contributed 1157 papers $(19.62 \%)$ and received 41,825 citations (30.97\%) during 1995-2018. Nine authors registered publication productivity above the group

Table 4: Subject-wise breakup of India's publications on nano-enabled drug delivery research during 1995-2018

\begin{tabular}{|c|c|c|c|c|c|c|c|c|c|}
\hline \multirow[t]{2}{*}{ Serial number } & \multirow[t]{2}{*}{ Subject* } & \multicolumn{3}{|c|}{ Number of papers (TP) } & \multicolumn{2}{|c|}{ Activity index } & \multirow{2}{*}{\multicolumn{3}{|c|}{$\begin{array}{l}\text { CPP TP (\%) } \\
1995-2018\end{array}$}} \\
\hline & & $1995-2006$ & 2007-2018 & $1995-2018$ & 1995-2006 & 2007-2018 & & & \\
\hline 1 & $\begin{array}{l}\text { Pharmacology, toxicology, and } \\
\text { pharmaceutics }\end{array}$ & 98 & 3070 & 3168 & 120.81 & 99.45 & 76,279 & 24.08 & 53.72 \\
\hline 2 & $\begin{array}{l}\text { Biochemistry, genetics, and } \\
\text { molecular biology }\end{array}$ & 35 & 1587 & 1622 & 84.27 & 100.41 & 44,066 & 27.17 & 27.51 \\
\hline 3 & Materials science & 23 & 1485 & 1508 & 59.56 & 101.06 & 37,273 & 24.72 & 25.57 \\
\hline 4 & Engineering & 14 & 1284 & 1298 & 42.12 & 101.52 & 23,431 & 18.05 & 22.01 \\
\hline 5 & Chemical engineering & 24 & 1208 & 1232 & 76.08 & 100.63 & 35,028 & 28.43 & 20.89 \\
\hline 6 & Medicine & 25 & 1125 & 1150 & 84.90 & 100.40 & 21,993 & 19.12 & 19.50 \\
\hline $\begin{array}{l}7 \\
\text { World output }\end{array}$ & Chemistry & $\begin{array}{l}25 \\
151\end{array}$ & $\begin{array}{c}1114 \\
5746\end{array}$ & $\begin{array}{l}1139 \\
5897\end{array}$ & 85.72 & 100.38 & 30,878 & 27.11 & 19.31 \\
\hline
\end{tabular}

There is overlapping of literature covered under various subjects. TP: Total paper, TC: Total citation, CPP: Citations per paper 
Table 5: List of significant keywords in Indian nano-Enabled drug delivery research literature, 1995-2018

\begin{tabular}{|c|c|c|c|c|c|c|c|c|}
\hline $\begin{array}{l}\text { Serial } \\
\text { number }\end{array}$ & Name of keyword & Frequency & $\begin{array}{c}\text { Serial } \\
\text { number }\end{array}$ & Name of keyword & Frequency & $\begin{array}{c}\text { Serial } \\
\text { number }\end{array}$ & Name of keyword & Frequency \\
\hline 1 & $\begin{array}{l}\text { Drug delivery } \\
\text { systems }\end{array}$ & 4349 & 37 & Nano-medicine & 430 & 73 & Biodegradability & 233 \\
\hline 2 & Nanoparticles & 3267 & 38 & Cell line tumor & 425 & 74 & Quantum dots & 217 \\
\hline 3 & Particle size & 2216 & 39 & Nanomaterial & 418 & 75 & Polyglycolic acid & 207 \\
\hline 4 & Drug delivery & 1607 & 40 & Lipids & 413 & 76 & Silver nanoparticles & 202 \\
\hline 5 & Drug formulation & 1572 & 41 & Drug penetration & 406 & 77 & Pegylate or PEG & 178 \\
\hline 6 & Drug release & 1479 & 42 & Drug distribution & 399 & 78 & Polyglyactin & 178 \\
\hline 7 & Drug carrier & 1472 & 43 & $\begin{array}{l}\text { Anti-neoplastic } \\
\text { activity }\end{array}$ & 395 & 79 & Polyglycolic acid & 177 \\
\hline 8 & In vitro study & 1469 & 44 & Polyglactin & 393 & 80 & Cyclodextrin & 172 \\
\hline 9 & Zeta potential & 1066 & 45 & Macrogol & 385 & 81 & $\begin{array}{l}\text { Polylactic acid-polyglycolic } \\
\text { acid copolymers }\end{array}$ & 165 \\
\hline 10 & Polymer & 986 & 46 & Drug absorption & 382 & 82 & $\begin{array}{l}\text { SiRNA or small interfering } \\
\text { RNA }\end{array}$ & 155 \\
\hline 11 & Nanotechnology & 896 & 47 & Surfactant & 382 & 83 & Polycaprolactone & 149 \\
\hline 12 & Drug solubility & 891 & 48 & Metal nanoparticles & 367 & 84 & Magnetic nanoparticles & 148 \\
\hline 13 & Liposomes & 872 & 49 & $\begin{array}{l}\text { Controlled drug } \\
\text { release }\end{array}$ & 361 & 85 & Chitin & 143 \\
\hline 14 & Nano bioavailability & 855 & 50 & Gold nanoparticles & 353 & 86 & Alginic acid & 126 \\
\hline 15 & Nano carrier & 817 & 51 & Dendrimer & 352 & 87 & Nanomagnetics & 118 \\
\hline 16 & Chitosan & 789 & 52 & Nanostructure & 345 & 88 & Polymeric nanoparticles & 112 \\
\hline 17 & In vivo study & 784 & 53 & Peptid & 334 & 89 & DNA & 108 \\
\hline 18 & Biocompatibility & 754 & 54 & Polymerization & 317 & 90 & Hyaluronic acid & 95 \\
\hline 19 & $\begin{array}{l}\text { Scanning electron } \\
\text { microscopy }\end{array}$ & 706 & 55 & Proteins & 316 & 91 & Colloid & 9 \\
\hline 20 & Nano-encapsulation & 680 & 56 & Conjugation & 316 & 92 & Iron oxide particles & 72 \\
\hline 21 & Encapsulation & 668 & 57 & Drug synthesis & 316 & 93 & Diagnostic imaging & 70 \\
\hline 22 & $\begin{array}{l}\text { Targeted drug } \\
\text { delivery }\end{array}$ & 650 & 58 & Curcumin & 313 & 94 & Metal nanoparticles & 70 \\
\hline 23 & Cytotoxicity & 642 & 59 & Pathology & 310 & 95 & Biodistribution & 67 \\
\hline 24 & Drug efficacy & 633 & 60 & Hydrophobicity & 306 & 96 & PLGA & 67 \\
\hline 25 & Doxorubicin & 616 & 61 & Nano pharmaceutics & 304 & 97 & Fluorescence imaging & 59 \\
\hline 26 & $\begin{array}{l}\text { Controlled drug } \\
\text { delivery }\end{array}$ & 607 & 62 & Microemulsions & 290 & 98 & $\begin{array}{l}\text { Superparamagnetic } \\
\text { nanoparticles }\end{array}$ & 59 \\
\hline 27 & $\begin{array}{l}\text { Solid-lipid } \\
\text { nanoparticles }\end{array}$ & 545 & 63 & Cancer therapy & 284 & 99 & $\begin{array}{l}\text { Semiconductor quantum } \\
\text { dots }\end{array}$ & 59 \\
\hline 28 & $\begin{array}{l}\text { Sustained drug } \\
\text { release }\end{array}$ & 538 & 64 & $\begin{array}{l}\text { Solid-lipid } \\
\text { nanoparticles }\end{array}$ & 250 & 100 & Ligand & 56 \\
\hline 29 & Nano-emulsion & 527 & 65 & Hydrogel & 282 & 101 & Polylactide & 47 \\
\hline 30 & $\begin{array}{l}\text { Solid-liquid } \\
\text { nanoparticles }\end{array}$ & 515 & 66 & Neoplasms & 276 & 102 & Polymeric miscelles & 38 \\
\hline 31 & $\begin{array}{l}\text { Differential scanning } \\
\text { caliometry }\end{array}$ & 492 & 67 & Nano encapsulation & 265 & 103 & Polypeptid & 37 \\
\hline 32 & Bioavailability & 487 & 68 & Nanofabrication & 264 & 104 & Copolymers & 26 \\
\hline 33 & Diseases & 462 & 69 & Breast cancer & 257 & 105 & Biomarkers & 26 \\
\hline 34 & Encapsulation & 458 & 70 & Carbon nanotubes & 254 & 106 & Fullerenes & 24 \\
\hline 35 & $\begin{array}{l}\text { Anti-neoplastic } \\
\text { agents }\end{array}$ & 434 & 71 & Glycol & 234 & 107 & Ceramics & 13 \\
\hline 36 & Paclitaxel & 434 & 72 & PEGs & 234 & 108 & Silica & 12 \\
\hline
\end{tabular}

PEGs: Polyethylene glycols

average of 57.85: N.K. Jain (109 papers), J.Al(84 papers), F.J. Ahmad (76 papers), S.P. Vyas (70 papers), R. Jayakumar (68 papers), S. Baboota (67 papers), A.K. Goyal (66 papers), O.P. Katare (60 papers), and B. Singh (59 papers). Six authors registered impact and relative citation index above the group average of $36.15 \mathrm{CPP}$ and 1.58: S.K. Sahoo (108.07 and 4.72), R.K. Khar (69.19 and 3.02), N.K. Jain (53.68 and 2.34), R. Jayakumar (49.29 and 2.15), A.K. Mishra (42.39 and 1.85), and J. Ali (37.64 and 1.64). Eleven authors registered ICP share more than the average ICP share $(18.15 \%)$ : P. Kesharwani (82.61\%), S. Beg Jamia (30.0\%), F.J.
Ahmad (27.63\%), A.K. Mishra (25.0\%), S. Jain (21.82\%), S. Baboota (20.90\%), R. Jayakumar (20.59\%), O.P. Katare (20.0\%), N.K. Jain (19.27\%), Z. Iqbal (18.92\%), and B. Singh (18.64\%) [Table 7].

Distribution of publications by source and channel of communication

Of the 5723 publications by India in Contribution and Impact research, $92.81 \%$ (3911) appeared in journals, 4. $41 \%(1440)$ as reviews, $1.49 \%$ (88) as conference proceedings, $1.03 \%$ (61) as book series and $0.25 \%(15)$ as 
Table 6: Scientometric profile of the top 20 most Indian productive organizations on nano-enabled drug delivery research during 1995-2018

\begin{tabular}{|c|c|c|c|c|c|c|c|}
\hline Serial number & Name of the organization & TP & $\mathrm{TC}$ & CPP & $\mathrm{HI}$ & $\operatorname{ICP}(\%)$ & $\mathrm{RCl}$ \\
\hline 1 & Jamia Hamdard University, Delhi & 304 & 7428 & 24.43 & 43 & $86(28.29)$ & 1.07 \\
\hline 2 & Dr Harisingh Gour University, Sagar, M.P & 278 & 10,792 & 38.82 & 56 & $41(14.75)$ & 1.70 \\
\hline 3 & Panjab University, Chandigarh & 169 & 3766 & 22.28 & 32 & $36(21.30)$ & 0.97 \\
\hline 4 & University of Delhi & 142 & 4315 & 30.39 & 31 & 46 (32.39) & 1.33 \\
\hline 5 & Indian Institute of Technology, Kharagpur & 113 & 4532 & 40.11 & 26 & 14 (12.39) & 1.75 \\
\hline 6 & Indian Institute of Technology, Mumbai & 112 & 1955 & 17.46 & 25 & $27(24.11)$ & 0.76 \\
\hline 7 & Banaras Hindu University, Varanasi & 107 & 3197 & 29.88 & 29 & $29(27.10)$ & 1.30 \\
\hline 8 & M. S. University of Varoda & 104 & 3763 & 36.18 & 34 & $12(11.54)$ & 1.58 \\
\hline 9 & Indo-Soviet Friendship College of Pharmacy & 104 & 1741 & 16.74 & 24 & $13(12.50)$ & 0.73 \\
\hline 10 & National Institute of Pharmaceutical Education and Research, Mohali & 101 & 7461 & 73.87 & 35 & $27(26.73)$ & 3.23 \\
\hline 11 & Central Drug Research Institute, Lucknow & 98 & 2174 & 22.18 & 23 & 19 (19.39) & 0.97 \\
\hline 12 & Indian Institute of Technology, BHU, Varanasi & 94 & 2369 & 25.20 & 26 & $21(22.34)$ & 1.10 \\
\hline 13 & Amrita Institute of Medical Sciences, Coimbatore & 93 & 3198 & 34.39 & 30 & $27(29.03)$ & 1.50 \\
\hline 14 & Institute of Nuclear Medicine and Allied Sciences, Delhi & 92 & 3091 & 33.60 & 32 & $15(16.30)$ & 1.47 \\
\hline 15 & Institute of Chemical Technology, Mumbai & 81 & 2784 & 34.37 & 23 & $14(17.28)$ & 1.50 \\
\hline 16 & Jadavpur University, Kolkata & 79 & 1010 & 12.78 & 20 & $9(11.39)$ & 0.56 \\
\hline 17 & Amrita Vishwa Vidyalayam University, Kochi & 79 & 2685 & 33.99 & 27 & $20(25.32)$ & 1.48 \\
\hline 18 & Indian Institute of Technology, New Delhi & 75 & 893 & 11.91 & 17 & $16(21.33)$ & 0.52 \\
\hline 10 & Indian Institute of Chemical Technology, Hyderabad & 68 & 1830 & 26.91 & 23 & $20(29.41)$ & 1.18 \\
\hline 20 & Indian Institute of Science, Bengaluru & 67 & 1345 & 20.07 & 22 & $26(38.81)$ & 0.88 \\
\hline Total & & 2360 & 70,329 & 29.80 & 28.9 & $518(21.95)$ & 1.30 \\
\hline \multicolumn{2}{|c|}{ Total of India } & 5897 & 135,043 & 22.90 & & & \\
\hline \multicolumn{2}{|c|}{ Share of top 20 organizations in India's total output } & 40.02 & 52.08 & & & & \\
\hline
\end{tabular}

TP: Total paper, TC: Total citation, CPP: Citations per paper, HI: h-index, ICP: International collaborative paper, RCI: Relative citation index

Table 7: Scientometric profile of top 20 most productive Indian authors in nano-enabled drug delivery research during 1995-2018

\begin{tabular}{|c|c|c|c|c|c|c|c|c|}
\hline Serial number & Name of the author & Affiliation of the author & TP & $\mathrm{TC}$ & CPP & $\mathrm{HI}$ & ICP (\%) & $\mathrm{RCl}$ \\
\hline 1 & N.K. Jain & Dr. Harisingh Gour University, Sagar & 109 & 5851 & 53.68 & 42 & $21(19.27)$ & 2.34 \\
\hline 2 & J. Ali & Jamia Hamdard University, Delhi & 84 & 3162 & 37.64 & 26 & $15(17.86)$ & 1.64 \\
\hline 3 & F.J. Ahmad & Jamia Hamdard University, Delhi & 76 & 2356 & 31.00 & 22 & $21(27.63)$ & 1.35 \\
\hline 4 & S.P. Vyas & IFS College of Pharmacy, Moga & 70 & 2177 & 31.10 & 29 & $3(4.29)$ & 1.36 \\
\hline 5 & R. Jayakumar & Amrita Vishwa Vidyalayam University, Kochi & 68 & 3352 & 49.29 & 27 & $14(20.59)$ & 2.15 \\
\hline 6 & S. Baboota & Jamia Hamdard University, Delhi & 67 & 2135 & 31.87 & 25 & $14(20.90)$ & 1.39 \\
\hline 7 & A.K. Goyal & Dr Harisingh Gour University & 66 & 1612 & 24.42 & 23 & $0(0.00)$ & 1.07 \\
\hline 8 & O.P. Katare & Panjab University, Chandigarh & 60 & 894 & 14.90 & 18 & $12(20.00)$ & 0.65 \\
\hline 9 & B. Singh & Panjab University, Chandigarh & 59 & 1012 & 17.15 & 18 & $11(18.64)$ & 0.75 \\
\hline 10 & S. Jain & $\begin{array}{l}\text { National Institute of Pharmaceutical Education } \\
\text { and Research, Mohali }\end{array}$ & 55 & 1677 & 30.49 & 21 & $12(21.82)$ & 1.33 \\
\hline 11 & S. Talegaonkar & Jamia Hamdard University, Delhi & 55 & 1988 & 36.15 & 19 & $5(9.09)$ & 1.58 \\
\hline 12 & G. Rath & IFS College of Pharmacy, Moga & 51 & 843 & 16.53 & 18 & $0(0.00)$ & 0.72 \\
\hline 13 & P. Kesharwani & Dr Harisingh Gour University, Sagar & 46 & 1357 & 29.50 & 18 & $38(82.61)$ & 1.29 \\
\hline 14 & S.K. Sahoo & Institute of Life Sciences, Bhubaneshw. & 45 & 4863 & 108.07 & 27 & $4(8.89)$ & 4.72 \\
\hline 15 & A.K. Mishra & $\begin{array}{l}\text { Institute of Nuclear Medicine and Allied } \\
\text { Sciences, Delhi }\end{array}$ & 44 & 1865 & 42.39 & 24 & $11(25.00)$ & 1.85 \\
\hline 16 & R.K. Khar & Dr. Harisingh Gour University, Sagar & 43 & 2975 & 69.19 & 22 & $5(11.63)$ & 3.02 \\
\hline 17 & T. Garg & IFS College of Pharmacy, Moga & 41 & 828 & 20.20 & 17 & $0(0.00)$ & 0.88 \\
\hline 18 & C.P. Sharma & SCTIMST, Trivandrum & 41 & 1414 & 34.49 & 19 & $5(12.20)$ & 1.51 \\
\hline 19 & S. Beg & Jamia Hamdard University, Delhi & 40 & 816 & 20.40 & 15 & $12(30.00)$ & 0.89 \\
\hline 20 & Z. lqbal & Jamia Hamdard University, Delhi & 37 & 648 & 17.51 & 13 & 7 (18.92) & 0.76 \\
\hline \multicolumn{3}{|c|}{ Total of 20 authors } & 1157 & 41,825 & 36.15 & 22.15 & $210(18.15)$ & 1.58 \\
\hline \multicolumn{3}{|c|}{ Total of India } & 5897 & 135,043 & 22.90 & & & \\
\hline \multicolumn{3}{|c|}{ Share of 20 authors in India's total } & 19.62 & 30.97 & & & & \\
\hline
\end{tabular}

TP: Total paper, TC: Total citation, CPP: Citations per paper, HI: h-index, ICP: International collaborative paper, RCI: Relative citation index

trade publications, and $1 \%$ as multivolume reference work during 1995-2018. Three hundred and seventy-six journals participated in Indian NEDD research, of which 282 journals contributed 1-10 papers each, 28 journals 11-20 papers each, 43 journals $21-50$ papers each, 19 journals 51-100 papers each, and 4 journals 101-169 papers each. The cumulative output of top 20 journals accounted for
$34.56 \%$ of total Indian journal output, which decreased from $40.35 \%$ during $1995-2006$ to $34.43 \%$ during 2007-2018. The leading journals contributing to Indian research in NEDD research are listed in Table 6. Colloid and Surfaces B. Biointerfaces (169 publications), followed by Nanomedicine (147 publications), International Journal of Macromolecules (156 publications), International 
Journal of Pharmaceutics (132 publications), etc., during 1995-2018 [Table 8].

\section{Highly cited papers}

Out of 5897 papers from the Indian scholars on NEDD research published during 1995-2018, there were 231 highly cited papers having received citations from 100 to 2802 citations per paper (153 in 100-200 citations range each, 49 in 200-399 citations range, 16 in 400-699 citations range, 3 in 700-100 citations range, and the remaining 7 papers in 1042-2802 citation range) during 1995-2018.

Together these 231 papers accounted for 58,559 citations, averaging 253.50 citations per paper. The 231 highly cited papers had participation from 20 countries: the USA (18 papers); Japan and Germany (7 papers); the UK, Italy, and Malaysia (4 papers each); France, Israel, Singapore, and South Korea (3 papers each); Brazil and Canada (2 papers each); and Austria, Belgium, China, Greece, Hungry, Ireland, Jordan, Norway, Oman, Portugal, Saudi Arabia, Slovenia, Serbia, South Africa, Spain, and Taiwan (1 paper each).

One hundred and eleven Indian organizations participating in these 231 highly cited papers, including Dr. H.S. Gour University, Sagar (27 papers), National Institute of Pharmaceutical Education and Research, Mohali and Institute of Life Sciences, Bhubaneswar (17 papers each), Jamia Hamdard University, Delhi (14 papers), M.S. University of Baroda (9 papers), University Institute of
Chemical Technology, Mumbai and Bombay College of Pharmacy (8 papers each), Postgraduate Institute of Medical Education and Research, Chandigarh (7 papers), University of Delhi, National Chemical Laboratory, Pune and Indian Institute of Technology, Kharagpur (6 papers each), SCTIMST, Trivandrum and Amrita Institute of Medical Sciences, Cochin (5 papers each), Karnatak University, Dharwad, Kakatiya University, Warangal, Pt. Ravishankar Shukla University, Raipur and Indian Institute of Chemical Technology, Hyderabad (4 papers each), National Institute of Interdisciplinary Research, Trivandrum, Central Drug Research Institute, Lucknow, INMAS-Delhi, National Institute of Science, Education and Research, Bhubaneswar, Indian Institute of Technology, Guwahati, Institute of Medical Sciences, BHU, Varanasi and J.S.S. College of Pharmacy, Ooctacamud (3 papers each), Sri Krishnadevaraya University, University of Kerala, Institute of Himalayan Bioresource Technology, Palampur, Bhabha Atomic Research Centre, Mumbai, Indian Institute of Technology, Mumbai, Birla Institute of Technology and Science, Pilani, Institute of Technology, BHU, Varanasi and Barchana's Women's College, Jaipur (2 papers), and rest of the organizations contributed only 1 paper each.

If the 231 highly cited papers (135 articles, 94 reviews, and 2 conference papers), 129 have zero collaboration and 102 involve national and international collaboration (64 national collaboratives and 38 international collaboratives).

Table 8: Top 15 most productive journals reporting output from Indian scholars in nano-enabled drug delivery research during 1995-2018

\begin{tabular}{|c|c|c|c|c|}
\hline \multirow[t]{2}{*}{ Serial number } & \multirow[t]{2}{*}{ Name of the journal } & \multicolumn{3}{|c|}{ Number of papers } \\
\hline & & 1995-2006 & 2007-2018 & $1995-2018$ \\
\hline 1 & Colloid and Surfaces B. Biointerfaces & 1 & 168 & 169 \\
\hline 2 & Nanomedicine & 1 & 146 & 147 \\
\hline 3 & International Journal of Macromolecules & 0 & 156 & 156 \\
\hline 4 & International Journal of Pharmaceutics & 9 & 123 & 132 \\
\hline 5 & Drug Delivery & 0 & 119 & 119 \\
\hline 6 & Journal of Biomedical Nanotechnology & 0 & 98 & 98 \\
\hline 7 & Journal of Drug Targeting & 8 & 86 & 94 \\
\hline 8 & Artificial Cells Nanomedicine and Biotechnology & 0 & 92 & 92 \\
\hline 9 & Expert Opinion on Drug Delivery & 0 & 86 & 86 \\
\hline 10 & Journal of Controlled Release & 8 & 74 & 82 \\
\hline 11 & Current Drug Delivery & 2 & 79 & 81 \\
\hline 12 & Materials Science and Engineering C & 0 & 79 & 79 \\
\hline 13 & RSC Advances & 0 & 77 & 77 \\
\hline 14 & Drug Development and Industrial Pharmacy & 3 & 72 & 75 \\
\hline 15 & International Journal of Pharmaceutical Sciences Review and Research & 0 & 74 & 74 \\
\hline 16 & International Journal of Pharmacy and Pharmaceutical Sciences & 0 & 72 & 72 \\
\hline 17 & Critical Reviews in Therapeutic Drug Carrier Research & 3 & 65 & 68 \\
\hline 18 & AAPS Pharmacitech & 6 & 61 & 67 \\
\hline 19 & International Journal of Nanomedicine & 1 & 63 & 64 \\
\hline 20 & Current Pharmaceutical Design & 4 & 55 & 59 \\
\hline \multicolumn{2}{|c|}{ Total of 20 journals } & 46 & 1845 & 1891 \\
\hline \multicolumn{2}{|c|}{ Total India journal output } & 114 & 5358 & 5472 \\
\hline \multicolumn{2}{|c|}{ Share of top 20 journals in Indian journal output } & 40.35 & 34.43 & 34.56 \\
\hline
\end{tabular}


The 231 highly cited papers were published in 88 journals, including 17 papers in Journal of Control Release, 16 papers in Nanomedicine, 11 papers in International Journal of Pharmaceutics and Biomaterials, 8 papers in European Journal of Pharmaceutical Sciences, 7 papers each in and European Advanced Drug Delivery Review, AAPS Pharm Sci Tech and Journal of Pharmaceutics and Biopharmaceutics, 5 papers each in Carbohydrate Polymers and Journal of Drug Targeting, 4 papers each in Colloids and Surfaces. B. Interfaces, Drug Discovery Today, Journal of Pharmacy and Pharmacology, Journal of Pharmacy and Pharmaceutical Sciences and Pharmaceutical Research, 3 papers each in Acta Biomaterialia, Biotechnological Advances, Bio-macromolecules, Chemical Society Reviews, International Journal of Nanomedicine, Journal of Antimicrobial Chemotherapy and Tuberculosis and 2 papers each in AAPS Journal, ACS Nano, ACS Applied Materials and Interfaces, Current Pharmaceutical Design, Biological and Pharmaceutical Bulletin, Critical Review in Therapeutic Drug Carrier System, Chemistry - A European Journal, Expert Opinion on Drug Delivery, Journal of Colloid and Interface Science, Journal of Pharmaceutical Sciences, Nanoscale and Polymer Plastic Technology and Engineering and 1 paper each in other journals.

\section{CONCLUSION}

There is a wide range of nanoparticulate materials and structures being developed for the delivery of therapeutic compounds. Each has its own particular advantages, but as these nanoparticles become optimized for their specific application, the outcome will be better-controlled therapy as a result of targeted delivery of smaller amounts of effective drugs to the required sites in the body. This is being made possible through the use of advanced material, improved control of particle size, and better understanding of interface between the biological and material surfaces and their effects in vivo. Some nanoparticle-based products are already approved by the USFDA, and several others are currently under development and clinical assessment. The analysis presented above offers useful technical intelligence to help researchers for identifying potential collaborators and to help inform $\mathrm{R}$ and $\mathrm{D}$ management and science and innovation policy for such nanotechnologies. Finally, it is suggested that India needs to develop specific plans of action and ensure its implementation at national level and also prioritize national goals for NEDD research.

\section{DISCUSSION}

The global NEDD research output $(53,079)$ originated in 134 countries during $1995-2018$. The $85.04 \%$ of total global publication share, however, came from only 10 most productive countries during 1995-2018, which showed increase from $82.08 \%$ during $1995-2006$ to $85.24 \%$ during 2007-2018. The USA, among top 10 countries, accounted for the highest publication share (24.95\%), followed by China (22.78\%), India (11.11\%), Germany, the UK, South Korea and Italy (from $4.00 \%$ and $4.58 \%$ ), and Iran, Japan, and France (from 3.46\% to 3.86\%) during 1995-2018. China, India, Iran, and Italy showed increase in their global publication share $(0.08 \%-115.28 \%)$, as against decrease in the USA, France, Germany, Japan. The UK and South Korea (from 3.46\% to 3.86\%) from 1995-06 to 2007-2018.

India had published 5897 publications in NEDD research during 1995-2018, which showed increase from 1 in the year 1995 to 95 in the year 2018, averaging $42.99 \%$ annual growth rate. India's share in the global publication output in NEDD research was $11.11 \%$ during 1995-2018, which showed increase from $4.52 \%$ during $1995-2006$ to $11.55 \%$ during 2007-2018. India's citation impact in NEDD research averaged to $22.90 \mathrm{CPP}$ during 1995-2018, which dropped from 150.66 during 1995-2006 to 19.24 during 2007-2018. The share of India's international collaborative papers was $20.35 \%$ during $1995-2018$, which showed increase from $14.57 \%$ during $1995-2006$ to $20.50 \%$ during 2007-2018. Pharmacology, toxicology and pharmaceutics registered the highest publications share $(53.72 \%)$, followed by biochemistry, genetics and molecular Biology $(27.51 \%)$, materials science $(25.57 \%)$, engineering $(22.01 \%)$, chemical engineering $(20.89 \%)$, medicine $(19.50 \%)$ and chemistry (19.31\%) during 1995-2018.

Six hundred and fifty-eight organizations and 811 authors participated in Indian NEDD research, of which the top 20 most leading organizations and authors together contributed $40.02 \%$ and $19.62 \%$ as their share of Indian publication output and $52.08 \%$ and $30.97 \%$ as their share of Indian citation output, respectively, during 1995-2018. Indian organizations, showing comparatively higher productivity in NEDD research were: Jamia Hamdard University, Delhi (304 papers); Dr. Harisingh Gour University, Sagar, M.P. (278 papers); Panjab University, Chandigarh (169 papers); and University of Delhi (142 papers). Similarly, Indian organizations showing comparatively higher citation impact per paper and relative citation index were: National Institute of Pharmaceutical Education and Research, Mohali (73.87 and 3.23), Indian Institute of Technology, Kharagpur (40.11 and 1.75), Dr Harisingh Gour University, Sagar, M.P. (38.82 and 1.70), M.S. University of Baroda (36.18 and 1.58), Amrita Institute of Medical Sciences, Coimbatore (34.39 and 1.50), Institute 
of Chemical Technology, Mumbai (34.37 and 1.50), Amrita Vishwa Vidyalayam University, Kochi (33.99 and 1.48), Institute of Nuclear Medicine and Allied Sciences, Delhi (33.60 and 1.47), University of Delhi (30.39 and 1.33) and Banaras Hindu University, Varanasi (29.88 and 1.30) during 1995-2018.

Indian authors showing comparatively higher productivity in NEDD research were: N.K. Jain (109 papers), J. Al (84 papers), F.J. Ahmad (76 papers), S.P. Vyas (70 papers), R. Jayakumar (68 papers), S. Baboota (67 papers), A.K. Goyal (66 papers, O.P. Katare (60 papers), and B. Singh (59 papers). Similarly, Indian authors showing with comparatively higher citation impact per paper and relative citation index were: S.K. Sahoo (108.07 and 4.72), R.K. Khar (69.19 and 3.02), N.K. Jain (53.68 and 2.34), R. Jayakumar (49.29 and 2.15), A.K. Mishra (42.39 and 1.85), and J. Ali (37.64 and 1.64) during 1995-2018.

Of the 5897 publications by India in NEDD research, $92.81 \%$ (3911) appeared in 376 journals. The 20 most productive journals contributed $27.31 \%$ share to the Indian journal publication output during 1995-2018. The publication shares from top 20 journals decreased from $40.35 \%$ during $1995-2006$ to $34.43 \%$ during $2007-2018$. The leading journals contributing to Indian research in NEDD: Colloid and Surfaces B. Biointerfaces (169 publications), followed by Nanomedicine (147 publications), International Journal of Macromolecules (156 publications), International Journal of Pharmaceutics (132 publications), etc., during 1995-2018.

Two hundred and thirty-one highly cited papers, out of output of 5897 publications on NEDD research from India, received from 100 to 2802 citations per paper during 1995-2018. These 231 highly cited papers together received 58,559 citations, averaging to 253.50 citations per paper. The USA accounted for the highest number of papers (18) in the 231 highly cited papers, followed Japan and Germany (7 papers); the UK, Italy, and Malaysia (4 papers each); France, Israel, Singapore, and South Korea (3 papers each); Brazil and Canada (2 papers each), etc., The 231 highly cited papers were published in 88 journals, including 17 papers in Journal of Control Release, 16 papers in Nanomedicine, 11 papers in International Journal of Pharmaceutics and Biomaterials, 8 papers in European Journal of Pharmaceutical Sciences, 7 papers each in Advanced Drug Delivery Review, AAPS Pharm Sci Tech and European Journal of Pharmaceutics and Biopharmaceutics, 5 papers each in Carbohydrate Polymers and Journal of Drug Targeting, and 4 papers each in Colloids and Surfaces. B. Interfaces, Drug Discovery Today, Journal of Pharmacy and Pharmacology, Journal of Pharmacy and Pharmaceutical Sciences and Pharmaceutical Research, etc.

\section{Financial support and sponsorship}

Nil.

\section{Conflicts of interest}

There are no conflicts of interest.

\section{REFERENCES}

1. Drug Delivery. Available from: https://en.wikipedia.org/wiki/ Drug_delivery. [Last accessed on 2019 Jan 21].

2. Huang Y, Ma J, Porter AL, Kwon S, Zhu D. Analyzing collaboration networks and developmental patterns of nano-enabled drug delivery (NEDD) for brain cancer. Beilstein J Nanotechnol 2015;6:1666-76.

3. Parveen S, Misra R, Sahoo SK. Nanoparticles: A boon to drug delivery, therapeutics, diagnostics and imaging. Nanomedicine 2012;8:147-66.

4. Robert C, Wilson CS, Venuta A, Ferrari M, Arreto CD. Evolution of the scientific literature on drug delivery: A 1974-2015 bibliometric study. J Control Release 2017;260:226-33.

5. Zhou X, Porter AL, Robinson DK, Shim MS, Guo Y. Nano-enabled drug delivery: A research profile. Nanomedicine 2014;10:889-96.

6. Yen-Chun L, Chen C, Xing-Tzu T. Visualizing the knowledge domain of nanoparticle drug delivery technologies: A scientometric review. Appl Sci 2016;6:1-14. 